The following is a chapter from

The Research Handbook on Organizational Resilience (2020)

Edited by Powley, E. H., Caza, B. and Caza, A.

ISBN: 9781788112208 


\title{
Organizational Resilience in Action: A Study of a Large-Scale Extended-Disaster Setting
}

\author{
Bernard Walker \\ Sanna Malinen \\ Venkataraman Nilakant \\ Katharina Naswall \\ Joana Kuntz
}

\begin{abstract}
"There's buildings down everywhere here. We need as many units as possible."
"We have got major damage. We have building collapses with people inside. We've got whole three-four storey complexes completely demolished."

"We're gonna need fire here now. We've got buildings on fire now as well."

(Police-radio communications immediately after the Christchurch earthquake)
\end{abstract}

A series of severe earthquakes rocked the Canterbury region in New Zealand from 2010 to 2016. One of the most destructive was a M6.3 tremor centred near the heart of Christchurch, the largest city in the region. This event was particularly violent, with some of the highest peak ground accelerations ever recorded for this type of earthquake. Unlike the Northridge, USA, and Kobe, Japan earthquakes, the tremor struck at midday on a workday, causing 185 fatalities and thousands of injuries (Ardagh \& Deely 2019). The effects were devastating, rating as the world's fifth-biggest insurance event at that time, with overwhelming damage to land, housing, physical infrastructure and businesses.

That M6.3 tremor was just the start of an extremely prolonged disaster situation. Destructive aftershocks continued for many months afterwards, with a series of large and late tremors not typical of other earthquakes (Christophersen, Rhoades, Hainzl , Smith, \& Gerstenberger, 2013). Much of the city's central business district remained closed for more than two years, and $70 \%$ of those buildings were demolished. Thousands of residents and businesses were displaced, with whole suburbs permanently abandoned, and major mental health challenges emerged among the population (Fergusson, Horwood, Boden, \& Mulder, 2014). Business operations were severely compromised and this was compounded by the ongoing tremors, with over 8,000 aftershocks in twelve months. The city's critical infrastructure networks of roads, water, waste, and power remained significantly impaired for an extended period. Agencies were confronted with a massive scale of urgent reconstruction work.

This was a complicated, extended-disaster setting, rather than a single event. It presented an extremely challenging environment for people and organisations, one that highlighted the paucity of evidence-based knowledge of resilience trajectories in relation to protracted adverse events. Earlier research into organisational resilience has tended to utilise either survey designs, or single organisations in a specific, time-defined disruption. In contrast, the Christchurch earthquake presented a rare opportunity for exploring organisational resilience in an extended crisis. It allowed our research team to study multiple organisations over a period of several years, as they simultaneously encountered wide-ranging disruptions, in a constantly changing environment. 
Our research programme involved an in-depth study of twelve lifeline ${ }^{1}$ organisations. The organisations were diverse, ranging from those managing physical infrastructure of water and roads, through to financial services and communication providers. They all shared a common feature; as lifeline organisations for the region, they had to continue functioning despite the disaster, providing essential services. The organisations had to manage multiple, competing demands in an unpredictable environment, with constrained resources, over an extended period. This provided an extreme test of their ongoing resilience.

\section{Resilience in the literature}

Recent decades have seen a growing interest in resilience, with writers exploring the concept at individual, group, and organisation levels. A diverse range of concepts are used, with resilience interchangeably referred to as either a trait, or state, or process variable (Britt, Shen, Sinclair, Grossman, \& Klieger, 2016; Estrada, Severt, \& Jiménez-Rodríguez, 2016; Norris, Stevens, Pfefferbaum, Wyche, \& Pfefferbaum, 2008; Sutcliffe \& Vogus, 2003).

Resilience can be viewed as a capacity that resides within an individual, team, or organisation, indicating a likelihood of positive adaptation. Writers such as Norris et al. (2008, p. 130) adopt a process-based view involving such capacities, defining resilience as "a process linking a set of adaptive capacities to a positive trajectory of functioning and adaptation after a disturbance." A similar process-based view of resilience has been presented by Williams et al. (2017), which describes resilience as a dynamic process, prior to and after an adverse event, where an individual, organization, or community interacts with the environment and utilizes various resources "in a way that positively adjusts and maintains functioning" (p. 742). Such a definition allows for resilience to be enacted at multiple levels, and for a protracted time, not just immediately following an event. The definition also suggests that there is positive adjustment, which may imply, but does not require, that there is post-adversity growth for resilience to be demonstrated.

There is growing interest in resilience at an organisation-level, framed as the ability of organisations, or organisational units, to positively adjust to adverse events (Powley, 2009; Sutcliffe \& Vogus, 2003). Within this, one perspective focuses on resilience as a personal characteristic of the employees in an organisation (Chen, M., \& Hobfoll, 2015; Vanhove, Herian, Perez, Harms, \& Lester, 2015; Varker \& Devilly, 2012). Other perspectives address the resilience of the organisation as an entity (Gittell, Cameron, Lim, \& Rivas, 2006; Lee, Vargo, \& Seville, 2013; Sutcliffe \& Vogus, 2003; van der Vegt, Essens, Wahlström, \& George, 2015). There is, however, only limited research addressing the interface between these perspectives. Recent research from an employee resilience perspective highlights the ways in which the organisational environment can influence employees' ability to be resilient at work, which in turn contributes to organisational resilience levels, acknowledging the person-environment interaction in resilience development (J.R.C. Kuntz, Malinen, \& Näswall, 2017; J. R C. Kuntz, Näswall, \& Malinen, 2016; Tonkin, Malinen, Näswall, \& Kuntz, 2018). The multilevel view of resilience presented by Williams et al. (2016) suggests that resilience can be enacted at different levels in the organization at the same time, and that resilience evolves and changes

\footnotetext{
${ }^{1}$ Defined as entities that provide essential infrastructure services to the community such as water, wastewater, transport, energy and telecommunications.
} 
over time as events unfold. Resilience, thus, does not only exist as a response to an event, but also as a capability an actor possesses prior to an event, and which affects how the actor reacts and copes (J. R C. Kuntz et al., 2016; Williams et al., 2017).

This employee-organisational resilience interface is an area of growing attention in the disaster recovery and resilience literature. Gittell et al. (2006) for example, explored organisational resilience in the USA airline industry following 9/11. Drawing on publicly available data, the researchers analysed elements such as stock prices, employee layoffs and strike action which were viewed as indicators of relationships, along with financial reserves, and business models. Based on the data, they proposed that an interplay between financial reserves and employee relationships contributed to organisational resilience. Powley (2009) examined an organisational crisis with a shooting and standoff, identifying social mechanisms that enabled healing among employees, and allowed the organisation to resume operations. From this, Powley proposed that the social connections, among and between organisational members, form a latent capacity that is activated in crises, enabling an organisation's resilience. Williams and Shepherd (2016) and O'Grady and Orton (2016) both report on the aftermath of the Haiti earthquake. Williams and Shepherd (2016) found that organizations created post-disaster to support recovery were vital to the progress, but only to the extent they were able to build and create resources based on what was available. O'Grady and Orton (2016) explore the way spirituality can support sensemaking and "cosmology episodes" in which an individual engages in a re-evaluation of their assumptions to fit the post-crisis context. By engaging in such re-evaluation and finding meaning in the new context, resilience is supported.

Research into organisation-level resilience links with the existing literature in areas such as organisational crises, adaptation, organisational learning, high performance and highreliability organisations, when these occur in dynamic and volatile environments (de Wall, 2012; Sutcliffe \& Vogus, 2003). As resilience reflects the organisations' ability to learn and adapt, a capacity for learning and transformation becomes relevant, linking with the extensive literature around organisational learning (for example Argote \& Miron-Spektor, 2011; Zollo \& Winter, 2002). Hence, organisational resilience is predicated on the extent to which organisations establish and develop learning cultures, including the processes of unlearning of established mindsets, values, beliefs and assumptions that may no longer be relevant.

Resilience and recovery are two interrelated but distinct concepts in the literature addressing disaster situations. Recovery is usually used to describe the process of a community, city, or organisation restoring to pre-disaster conditions after a major crisis (Aldrich, 2012). Recovery is often protracted and can vary among different groups experiencing the same crisis. Resilience is the capacity to engage in the recovery process (Aldrich, 2012). More resilient individuals, organizations, and communities are better able to utilize and create resources that facilitate recovery, thus adapting more successfully to the post-crisis environment (cf. Williams \& Shepherd, 2016).

\section{Our research programme}

Our research drew on Britt et al.'s (2016) definition of resilience, involving the demonstration of positive adaptation in the face of significant adversity, which involves not only bouncing 
back, but also growth or positive changes. As this was a sudden-onset disaster, characterised by fast, discontinuous changes, it required a series of very rapid adaptations, which differentiate it from the progressive change typically involved in traditional, business survival literature (Bhamra, Dani, \& Burnard, 2011).

The Christchurch earthquake setting clearly met the threshold for a significantly adverse event triggering a complex resilience response (Britt et al., 2016). It involved a large-scale disaster, with extensive impact on the built, economic, social, and natural environments. Organisations were operating in a context of ongoing uncertainty, as they confronted a series of major challenges to their business functions. The wider backdrop involved a distressed community, where the disaster had major effects on the health and well-being of individuals and groups (Johal, Mounsey, Brannelly, \& Johnston, 2016).

The focus of our research was on the resilience of the organisations. Two complementary perspectives were utilised. One was organisational resilience, viewed as a process that enables an organisation as a whole to demonstrate positive adjustment and adaptation (Sutcliffe \& Vogus, 2003; Vogus \& Sutcliffe, 2007). In this view, organisational resilience is not simply the sum of the individual resilience of organisational members; rather, it is the functioning of the organisation as an entity.

The other perspective was that of employee resilience, defined as "behavioural capability to leverage work resources in order to ensure continual adaptation, well-being, and growth at work, supported by the organization" (J.R.C. Kuntz et al., 2017, p. 224). Rather than viewing resilience as primarily the responsibility of individuals, this view posits resilience as a capability that is developed through the culture, work practices and leadership styles in the organisation. These factors influence whether employees are resilient, signalled by their capacity to learn, adapt to change, collaborate, and generate resources at work.

\section{Research methods in a disaster setting}

Ideally, longitudinal designs, with baseline and follow-up measures, allow researchers to examine how different agents adapt to adversity over time, in order to capture 'resilience trajectories' (Britt et al., 2016). In practice, however, large-scale adverse events such as sudden-onset disasters cannot be predicted in advance. This reduces the ability to assemble pre- and post-event data sets, but it does provide a unique laboratory in which to explore behaviour and change through a different lens (Britt et al., 2016; Hall et al., 2016). Our programme explored the experiences of a range of organisations, as they simultaneously encountered the common crises that emerged.

Our research methodology was based on identifying cases that were evidenced as showing resilience, then searching for differentiating factors. However, as the Christchurch situation involved an ongoing, unfolding and uncertain set of circumstances, the methods had to be adaptive, allowing for unanticipated changes within organisations or in the physical environment. Post-disaster research has many challenges; a sudden, major aftershock for example, would close down access. This meant that pragmatic research approaches were needed. For example, although the ideal would have been a sequential progression where qualitative data gathering and analysis were largely completed before moving to the 
quantitative phase, there was a need to capture data while access was available and before recall bias affected responses (Hall et al., 2016). This also had to be balanced against the fact that the situation was continuously evolving, and ceasing data gathering too early would have omitted significant developments.

The study adopted a mixed-methods approach conducted by a multidisciplinary research team. The case study design (Eisenhardt \& Graebner, 2007; Stake, 2000; Yin, 2003), used paired organizations within an industry sector, with a total of eleven organisations studied indepth.

Table 1. Participating organisations by industry and size.

\begin{tabular}{|l|l|l|l|}
\hline Organisation & Industry sector & Employees & Infrastructure Sector \\
\hline A & Construction & $1000-4000$ & $\begin{array}{l}\text { Water and waste; roading, landcare, } \\
\text { buildings provider }\end{array}$ \\
\hline B & Energy & $300-500$ & Electricity supplies and distribution \\
\hline C & Transport & $500-1000$ & Key transport-gateway provider \\
\hline D & Transport & $<500$ & Key transport-systems provider \\
\hline E & Communications & $4000+$ & Telecommunications provider \\
\hline F & Local authority & $300-500$ & Local government organisation \\
\hline G & Local authority & $1000-4000$ & Local government organisation \\
\hline H & Finance & $4000+$ NZ & $\begin{array}{l}\text { Banking and financial-services } \\
\text { provider }\end{array}$ \\
\hline I & Finance & $4000+$ NZ & $\begin{array}{l}\text { Banking and financial-services } \\
\text { provider }\end{array}$ \\
\hline J & Construction & $4000+$ NZ & $\begin{array}{l}\text { Roading, transport infrastructure, and } \\
\text { utilities provider }\end{array}$ \\
\hline K & Construction & $\begin{array}{l}\text { Roading, transport infrastructure, and } \\
\text { utilities provider }\end{array}$ \\
\hline
\end{tabular}

The qualitative section involved one-to-one, in-depth interviews and focus groups. Typically, 15 participants were interviewed from each organization, although this varied with organisation size, drawing on personnel from a range of roles and sectors across each organisation. A total of 167 individuals participated. Key informants were interviewed more than once to further explore emerging themes, producing a total of over 200 interviews. The majority of the individual participants were supervisors and middle or senior managers, and the balance were frontline workers. 
Table 2. Interview participants by industry sector.

\begin{tabular}{|l|c|}
\hline \multicolumn{1}{|c|}{ Industry sector } & Number of interviewees \\
\hline Construction & 48 \\
\hline Energy \& Communication Transmission & 28 \\
\hline Transport & 26 \\
\hline Local authorities & 27 \\
\hline Finance & 38 \\
\hline
\end{tabular}

As the phenomenon being studied was relatively unchartered and dynamic, emergent, inductive and open-ended methods were most appropriate. The transcribed data were analysed using grounded procedures. This involved open and focused coding, constant comparison, and theoretical memos (Bryant \& Charmaz, 2007; Charmaz, 2008; Strauss \& Corbin, 1997). Throughout the project, the research team compared and discussed open codes, and emerging theoretical concepts. From this, detailed within-case analyses were created which were then used in cross-case analyses to explore the ways in which key concepts functioned. Extant literature and theoretical perspectives were drawn upon throughout the research process (Charmaz, 2014).

Accompanying this, quantitative surveys were conducted in a selection of organisations that participated in the interviews, with the surveys accessing larger samples of organisation members. The surveys measured constructs that emerged from our qualitative investigations. The results from the quantitative surveys are reported on in more detail in Näswall et al. (2017).

The research approach captured the progress of the organisations through the disaster and recovery, focusing on the three-year period following the initial, major seismic incident. Figure 1 presents the timeline of our research. Data collection commenced eight months after that major tremor. This allowed time to move beyond the initial state of civil emergency and after an initial moratorium on research, as well as permitting the researchers to negotiate access and obtain ethics approvals. As the extended three year timeframe involved ongoing, rapid adaptation to major disruptions, the research was able to produce findings that approximated resilience trajectories, with the development and progression of an organisation's resilience. The aim was to produce a comprehensive, grounded account of resilience-in-action during a large-scale, extended disaster.

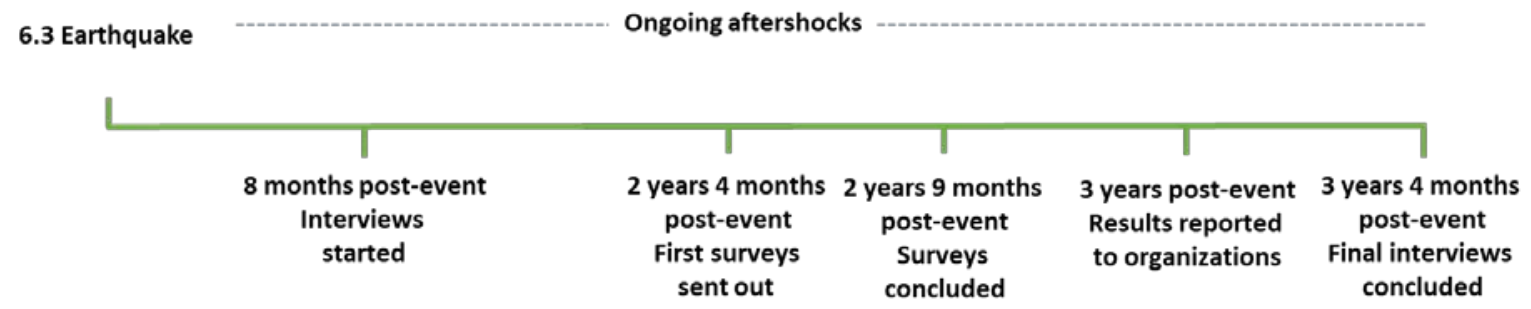

Figure 1. Timeline of major events and research milestones 


\section{The Organisations}

The lifeline organisations studied were providers of water and waste systems, road and air transport, energy supply lines, local government, financial services, and telecommunications.

One set of the organisations dealt with the physical infrastructure. These organisations faced a massive challenge; $89 \%$ of the city's residential dwellings, hundreds of kilometres of underground water and waste pipes, hundreds of pump stations, bridges and reservoirs, much of the power network, and over a million square metres of roadway, were all in need of urgent repair. For those organisations, there was a tension between speed and sustainability. Their work was of great urgency for the city's recovery, so there was a need for long hours and intensive work, but the scale of damage meant that this work would continue for months or years. They had to learn ways of working, often with large numbers of projects, personnel and resources, to achieve high levels of output, while at the same time being sustainable in the longer term.

The second set of organisations were not dealing directly with the physical restoration of the city, but they were also functioning in a new environment and faced significant challenges. Even for sectors such as financial service providers, the major physical and population changes meant that customer-flows were radically redistributed. As a result, employees encountered resourcing issues, as some sites were overloaded with work, while others had significantly reduced volumes. The emotional demands of the work changed. Client-facing interactions were often highly emotive, as residents showed significant signs of stress and trauma. The staff too were dealing with their own significant physical and psychological stressors.

\section{What did adaptation look like?}

Unlike Powley's (2009) case involving a single-episode, organisation-specific incident, these lifeline organisations did not have a 'temporary holding space' where work was suspended. Individuals and organisations were rapidly thrust into volatile, unfamiliar territory. Their prewritten Business Continuity Plans (BCPs) provided little guidance in the ongoing situation, as they never anticipated the extent of damage, nor the duration of the crisis.

Significant disruption occurred at three levels. At the organisational level, the organisations' facilities, IT and communication systems, were damaged or destroyed. Large inflows and outflows of staff occurred. Traumatised staff abandoned the city. Other workers and equipment arrived from outside the region, sometimes more than doubling the workforce. The supplies of resources were often inadequate. An electricity supply organisation, for example, normally held a reserve stock of cable joint-kits for reconnecting broken lines. After only eight days, they had exhausted the entire available supply in two countries, Australia and New Zealand. 
The personal-level disruption was also substantial, as staff dealt with injuries or bereavements, damage to their homes and property, along with anxiety and stress from the ongoing tremors. In the accompanying societal-level disruption, access to shops, fuel supplies, schools and public transport were affected, while many homes lacked the basics of water, waste, and power.

Overall, this produced an unknown and unpredictable situation that required rapid responses. Adaptation meant having to improvise in settings where there were few guidelines, and the consequences of any decisions were largely unknown. Analysis showed that this adaptation clustered around four, interdependent factors; leadership and situational awareness, employee-centric orientation, collaboration, and learning. These factors were already present in varying degrees in the organisations, and continued to develop during the extended crisis.

\section{Leadership}

The discussion commences with leadership, as this was a critical element that distinguished the more resilient organisations. Typically, the leader's personal style and attributes were echoed in the culture of the wider organisation.

Across the organisations that showed greater adaptation to their situation, there was a common pattern of approachable, empathetic and people-oriented leaders. One CEO, for example, hired a mobile coffee shop and went around the many, tired work crews doing roadrepair work, talking with them, and making them feel valued and involved. These leaders were accessible to the workers, providing tangible support and recognition of the workers' efforts;

It probably cost us $\$ 30,000, \$ 40,000$ a week having that coffee van out there...but just the value of that in terms of engagement from our people was incredible.

These leaders exhibited genuine concern for the workers, could understand their perspectives and challenges, and reached out to staff. They symbolically and verbally communicated a value system to the rest of the organisation, including the importance of caring for workers, giving direction, purpose, support and acknowledgement. Although they were what one interviewee referred to as "blokes' blokes", they were clearly empathetic leaders (Boyatzis, Goleman, \& Rhee, 2000; Boyatzis \& McKee, 2005).

They just wanted to spend time with staff...I can see him, looking at his face, what he was worried about was our staff.

In contrast, in the organisations that had greater problems with adapting, the senior managers remained more hierarchical and remote, focusing on operational matters ${ }^{2}$. They were often based outside the region and in some cases they never visited the affected work sites. They showed poor understanding of the post-disaster situation, the resourcing, and the workers' needs. Workers consequently felt unappreciated and unheard, resulting in

\footnotetext{
${ }^{2}$ Most organisations addressed the operational matters thoroughly but the differentiating factor was that the leaders in the more adaptive ones also focused on the human side of the situation.
} 
resentment, lack of engagement and being less willing to expend discretionary effort. Often these organisations lacked a unifying vision.

In the more resilient organisations, the senior leaders simultaneously addressed operational and strategic issues. They took ownership of the situation, provided leadership in their areas, and were prepared to take risks with initiatives. The CEOs typically created a united, senior leadership team around them, with a shared sense of ownership and shared values. They empowered that team to actively deal with matters, especially crisis-related operational issues, so that the CEO could often step back and deal with more strategic aspects.

These executive-level leaders united the organisation, providing a shared identity and vision. They articulated a common purpose for all the exhausting work effort, shaping this in terms of serving the wider community in a time of crisis. The leaders interviewed showed an openness to learning in their own lives, including gathering input from other people.

This leadership, at middle and senior levels, supported the other key factors that accounted for organisational resilience: leaders' situational awareness, an employee-centric orientation, collaboration, and learning.

\section{Situational awareness of Leaders and Organisations}

Conventional wisdom suggests that, in the aftermath of a natural disaster, organisational leaders would be aware of significant changes to the work context, scanning for new and emerging issues. This was not the case across all the organisations surveyed though. Situational awareness emerged as a critical feature that differentiated between the organisations in terms of their adaptation to the crisis environment. It refers to the ability of leaders to perceive arising changes and interpret novel situations, to proactively identify important issues, and then aptly respond to them.

So, I was quite conscious in my own mind about styles because they change and as we've worked through into recovery, the approach required in recovery, and I think this is a learning across all of Canterbury is, that an entirely different mindset is required to manage recovery versus response. So, it was just a self-awareness thing really. Situational. You would just call it situational management. Identifying what the issues are and then working out how to get the best out of people and the best approach to take

Leaders in the more adaptive organisations had a higher level of situational awareness. They accurately appraised novel and unique situational features and were able to prioritise action accordingly. This was particularly manifested in their approach to employee wellbeing, internal and external collaboration, and organisational learning. Situational awareness encompassed mindfulness and mindful caring (Powley, 2009), noticing and understanding the perspective of workers, leading to empathy.

In the organisations that adapted well, senior executives were not only alert to emerging issues themselves, but were also active in fostering ongoing situational awareness as part of the organisation's routines. They empowered other leaders to address specific issues, linked 
to their unique area of expertise. For example, some senior executives appointed second-tier leaders better attuned to staff needs, to maintain an ongoing, active focus on issues such as wellbeing. This further developed the situational awareness of the overall organisation.

\section{An employee-centric orientation}

In the first weeks after the earthquake, all the organisations responded in a similar manner providing for immediate, tangible staff needs. They offered support such as hotel showers, food vouchers, water supplies, and even emergency house repairs. This mirrored a wave of support occurring in the wider community. However, employee needs persisted throughout the longer timeframe of the extended crisis, and evolved toward more subtle psychosocial risks, such as mental health issues, fatigue, and ill-health symptoms. Yet, not all organisations were alert to these employee dynamics (Nilakant, Walker, Rochford, \& van Heugten, 2013).

The extent to which organisations were aware of employees' needs and wellbeing in the medium to long term constituted a second differentiating factor. In the more adaptive organisations, staff wellbeing remained one of their priorities throughout the crisis. Those organisations mirrored Powley's (2009) notion of 'carefully mindful', remaining in touch with staff perspectives, acknowledging, and offering support for challenges, and in these ways fostering resilience activation. By comparison, the less adaptive organisations lacked this type of mindfulness, and were less aware of, and less responsive to, staff wellbeing issues.

This employee-centric orientation fostered the types of relationships, and the alignment between workers and management, that facilitated higher levels of adaptation. The emphasis on employee wellbeing reflected a broader organisation-wide culture, and a people-focused way of working. The more employee-centric organisations were characterised by positive workplace relationships, with genuine care and respect for others. Importantly, these ways of working existed prior to the disaster, and the support for wellbeing that was offered during the extended crisis was just symptomatic of a longstanding and well-established employeecentric orientation.

Middle managers were a key element in this employee-centric way of working. In the more adaptive organisations, middle managers were treated as valuable partners. They shared this employee-centric orientation, clearly seeing employee support as part of their role; they were empathetic, and had the capabilities to perform this function. These managers typically felt they were listened to, and connected to senior leaders, as well as to their staff.

In one very successful organisation, almost every manager counted this people-focus as one of the most important aspects of their role. Their empathetic concern extended to employees' psychological wellbeing, and understanding the disaster-related pressures outside of work. These managers were also attuned to individual differences, rather than applying "one-sizefits-all" solutions.

So I think that's where the hierarchy was really strong. So we had twice daily conference calls with our Store Managers who were in touch with all their staff and the whole call was about how are your staff, what do they need and everything that they needed we just got for them. It has been really a testament to our engagement 
of our staff now. They are still blown away by what we did for them over a long period.

In contrast, with organisations that did not have an employee-centric orientation, local managers were less likely to be empowered to address issues such as staff wellbeing, and if this did finally happen, it tended to be much later in the crisis. Some local middle managers lacked people management capabilities, and were slow to respond to problems affecting staff. They also had a lesser sense of togetherness and alignment with their seniors. The organisations that encountered people-related problems were typically cases where the managers tended to pay only lip-service to staff support. In those organisations, even when HR staff offered a number of supports, there was little uptake. Instead, their staff became dissatisfied, and a range of problems persisted in those organisations.

In other instances, the middle managers themselves were more empathetic, but had to work in an unsupportive, wider organisational culture. While these managers sought to counter that organisational culture, they were often forced to implement non-supportive practices that they personally did not believe in, but were unable to challenge. This had a detrimental effect on their own wellbeing, including perceptions of high emotional labour, stress, and reports of poor psychological and physical wellbeing. In one organisation, middle managers simply ignored some of the senior leader directives, as it was felt that staff concerns were not heard or acted upon.

Over the duration of the crisis, the contrast between the employee-centric organisations, and those that did not display such an orientation, continued to magnify. The resilience trajectories were markedly different. The employee-centric orientation of the more adaptive organisations, existed before the disaster, in the mindsets, the routines and the capabilities in the organisation. This orientation gave them a greater situational awareness regarding staff-related issues, and allowed them to respond more fully to those demands in the new situation. The capacity was utilised from the outset of the disaster, and continued to develop further over the duration. In contrast, the less adaptive organisations had a different trajectory. They belatedly discovered the problems caused by their existing approach, and then began to create this capacity much later in the disaster. In some situations, the move towards more people-centric policies and culture only occurred years after the initial significant quake, after coming to realise that a results-oriented focus, at the expense of people orientation, was costly and ineffective in the post-disaster context.

\section{Collaboration and Networking}

The third factor that differentiated between organisations with regard to their resilience trajectories was the ability to leverage collaborations. Despite the major constraints and resource shortages, some organisations excelled, achieving significant outcomes in short timeframes. This reflected the network of relationships that they were able to draw upon, both internally and externally.

Externally, these organisations possessed a range of relationships that were often established prior to the crisis. This provided them with external partners that were willing and able to 
help from the very outset of the crisis. Their speed in obtaining support was a major asset in a time-critical setting.

When the electricity provider mentioned earlier, had exhausted all the supply of cable jointkits in the Southern Pacific area, they drew upon their relationships and networks. They arranged with a German manufacturer to do a special production each day and fly them across the world, so that at daybreak each morning a new supply would arrive in Christchurch for the work teams. That process was repeated daily through the recovery.

Germany were making fault kits as fast as we could use them. So there was this huge supply chain just to get the jointing kits in that were needed to actually keep repairing. When you think of it, there was a whole world out there supporting a rebuild or just getting on with the rebuild, just getting it connected.

These networks were a central part of these organisations' way of working, and they were skilled at developing and maintaining relationships. In some situations, the disaster disrupted the pre-existing networks. Although 'swift trust' did emerge in a few instances, the predominant pattern was that when new connections were needed, these organisations had the ability to create them (Beck \& Plowman, 2014). They tended to make this a deliberate, high-priority activity. In several instances, the CEO took on role of liaising with external organisations. In other cases, it was devolved to a team. One team, for example, developed and managed a map of their networks, identifying all the key organisations that they needed to either strengthen existing collaboration with, or initiate a new relationship.

Internal collaboration was also important. When senior leadership teams worked closely together, that collaboration was typically accompanied by collaboration across the functional areas of the organisation. Well-developed collaborative relationships with other geographical units allowed the local units to draw on much needed staff and equipment resources.

In being able to access extra resources, equipment, and people, these organisations could share the vast task with a range of other groups and agencies, covering complementary areas. They could access their networks for additional insight and technical support, and could enter into joint projects. These connections fostered their innovation and gave them legitimacy that allowed them to take new initiatives (Beck \& Plowman, 2014).

This contrasted with the other organisations without the same connections and experience. In some instances, the senior executives of those organisations looked back over their experience and observed that their organisations would have adapted much better if they had collaborated. Networking was not a central part of their mindset and normal routines though, and therefore was not prioritised.

I don't think it was necessarily a matter of 'no way, we're not working with those guys'. It's probably just more a matter of maybe none of us had formal working links with leadership of other [industry] and to work out how we can support each other.

Again, these factors influenced resilience trajectories. Some organisations belatedly began to develop networks during the extended crisis. Similarly, organisations that lacked internal 
collaboration gradually discovered that they needed to develop this during the extended crisis, moving away from the isolated ways of working. These behaviour patterns produced slower trajectories of resilience development.

\section{Collective Learning}

Prior to this disaster, most organisations had only experienced short-term crises lasting a few days. After those temporary disruptions they returned to business-as-usual. In that type of situation, learning simply involved retrospective reviews once the disruption had ended, as a process of learning-from-disaster. The Christchurch extended disaster situation however, was vastly different, with ongoing disruptions, and no foreseeable end-point or return to their normal situation. It involved months or years operating in an unknown setting. This required a dynamic process of rapidly learning-in-disaster, adapting to new situations as they emerged. The capacity for this ongoing, real-time learning and adaptation constituted the fourth key differentiating factor in terms of the organisations' adaptation.

Learning depended on organisations perceiving the need for improvement, and having the capacity to act on this. Several months before the major city quake, the city had a preview of a major seismic event, with a tremor centred outside the city, but without major city destruction. For organisations such as a vital transport access-point, this was their first major earthquake. Their limited preparation and experienced hampered their response, as they scrambled to implement back-up systems and processes for re-opening their facilities. As an essential access lifeline, their delays had major consequences for the city.

After the incident, the management team set up a process to learn from the events and devise systems for handling seismic incidents. A number of other organisations shared this approach, viewing the events as an opportunity for learning and change;

...event gave us this massive opportunity to take everything that we had learnt from the first one, everything we thought that we screwed up, maybe not quite everything, and do it differently.

When the devastating city-based quake occurred a few months later, the transport access point put their improved systems into practice, re-opening access to the city in a much shorter timeframe. They calculated what those improvements meant, in terms of the numbers of outside rescuers brought into the city with the earlier re-opening, the number of rescues performed and lives saved, in that time. These types of successes boosted the organisation's motivation for ongoing learning, with a new sense of efficacy and a readiness to tackle other challenges (Sutcliffe \& Vogus, 2003).

So we always debrief. You can say anything you like and it's got to the point now where we go 'I could have done this better' and we go 'right, what do we need to do that'. It becomes almost a blameless environment and people are very open.

Other organisations did not initially perceive a need for change though, until after the devastating city quake, when they encountered a complex and unfamiliar situation where their previous experience offered little guidance. For all the organisations, there was a tension 
between the known and unknown throughout the disaster situation. Their observable activities often remained largely the same, working on roads, laying power cables, or coordinating transport, however the context had radically changed. The organisations that remained focused on their existing routines, but paid less attention to the changed context, failed to fully recognise the learning needed for change and adaptation.

Organisations such as the transport access agency had a higher level of situational awareness, perceiving that need for change and learning, and they also had the capacity to implement this. Even before the disaster, they valued staff input, and were open change. Those organisations remained flexible and innovative, continuously adapting to emerging issues and developing new ways to work. Some organisations moved beyond single loop learning. They produced new technical processes, new organisational systems and processes, and acquired new equipment and expertise; in addition, they went on to develop major changes in their ways of thinking (Wang \& Ahmed, 2003). Significantly, they also learned how to keep on learning.

Several organisations actively translated their recent disaster-related insights into ongoing, new ways of working that continued after the disaster, including new empowered leadership, simpler systems for faster decision making, and structures that were more responsive to local issues;

So [name] has gone in and she has taken 20 steps out of a 30 step process, got it down to 10 steps and they go 'oh are we allowed to do it that way?' and we go 'yeah'.

The resilience trajectories of those learning-oriented organisations were enabled by the other three key elements of our proposed resilience framework. Their employee-centric orientation fostered a supportive learning environment and psychological safety, where members could experiment and risk failures. Their collaboration set the foundation for collective learning, information sharing and joint exploration. Their leadership reinforced a culture of learning throughout the organisation. During the disaster, those capacities developed further, as part of a virtuous cycle, promoting their ongoing adaptation (Sutcliffe \& Vogus, 2003).

In contrast, less resilient organisations tended to perpetuate their pre-disaster routines, with less openness to taking on new information or unlearning ineffective practices. In one organisation, for example, the senior leaders were located outside of the region, and lacked understanding of the Christchurch situation. They required that staff rigidly follow an existing set of disaster procedures that had been developed from another location, from a different type of disaster. Those procedures did not match the extended seismic setting. This adversely affected the relationship between the workers and management, causing frustration and stress as the organisation's systems were out of alignment with the changed context, especially the nature and volumes of the new work environment.

Once again, the organisations that lacked the capacity for learning showed much slower adaptation and growth. It was only much later in disaster, when the consequences of their rigid systems and mindsets became evident, that they belatedly realised the need for change. At that point they were still limited by the extent to which their organisational 
environments had the capacity to support learning, producing slower resilience trajectories.

Although sub-units sometimes attempted to develop their own learning, the unsupportive wider organisational culture effectively obstructed new ways of working. Compounding this, when an organisation's sub-units functioned in isolation from each other and did not share new knowledge, there was even less chance of collective learning. Individual staff sometimes gained new knowledge from working alongside other organisations, but if their own home organisations were not open to learning, and lacked systems for gathering and implementing new insights, this became a potent source of frustration and disillusionment for those staff.

\section{What can be learned from the research?}

The findings of this research give rise to a model of how organisational resilience is socially enacted, and significantly, how it continues to develop, during an extended-disaster. The model is based around a set of capacities: leadership, employee-centric orientation, collaboration, and learning. It affirms how these are largely relational, involving social processes, with the organisation as a whole going through revision of its cosmology, adapting its worldview to incorporate the post-crisis context (Gittell et al., 2006; Powley, 2009). These capacities function as resources that are utilised in a crisis, enabling the organisation to adapt.

An important contribution from this research is the insight into the interdependency between the four key capacities, and more specifically, how they function as a developmental sequence. Figure 2 presents these as a maturity model with key areas. The starting point of the resilience process at Level 1 , is creating a foundation of an employee-centric orientation where employees are valued, promoting trust and engagement. Without this basis, the other levels are not possible to enact. From there, when employees are supported, they can engage in collaboration and resource sharing, both within and outside their immediate unit (Level 2). Ultimately, from these bases, the organisation can progress to the organisational learning and sharing of knowledge (Level 3).

Leadership has a key, ongoing role, through each of the stages, influencing each of the other capacities. Leaders of resilient organisations exhibit the three core capacities in their own lives, and simultaneously promote their development throughout the organisation. The leaders' own personal capabilities enable them to have high levels of situational awareness, perceiving the needs in each of the key areas, and acting in ways to foster resilience. 


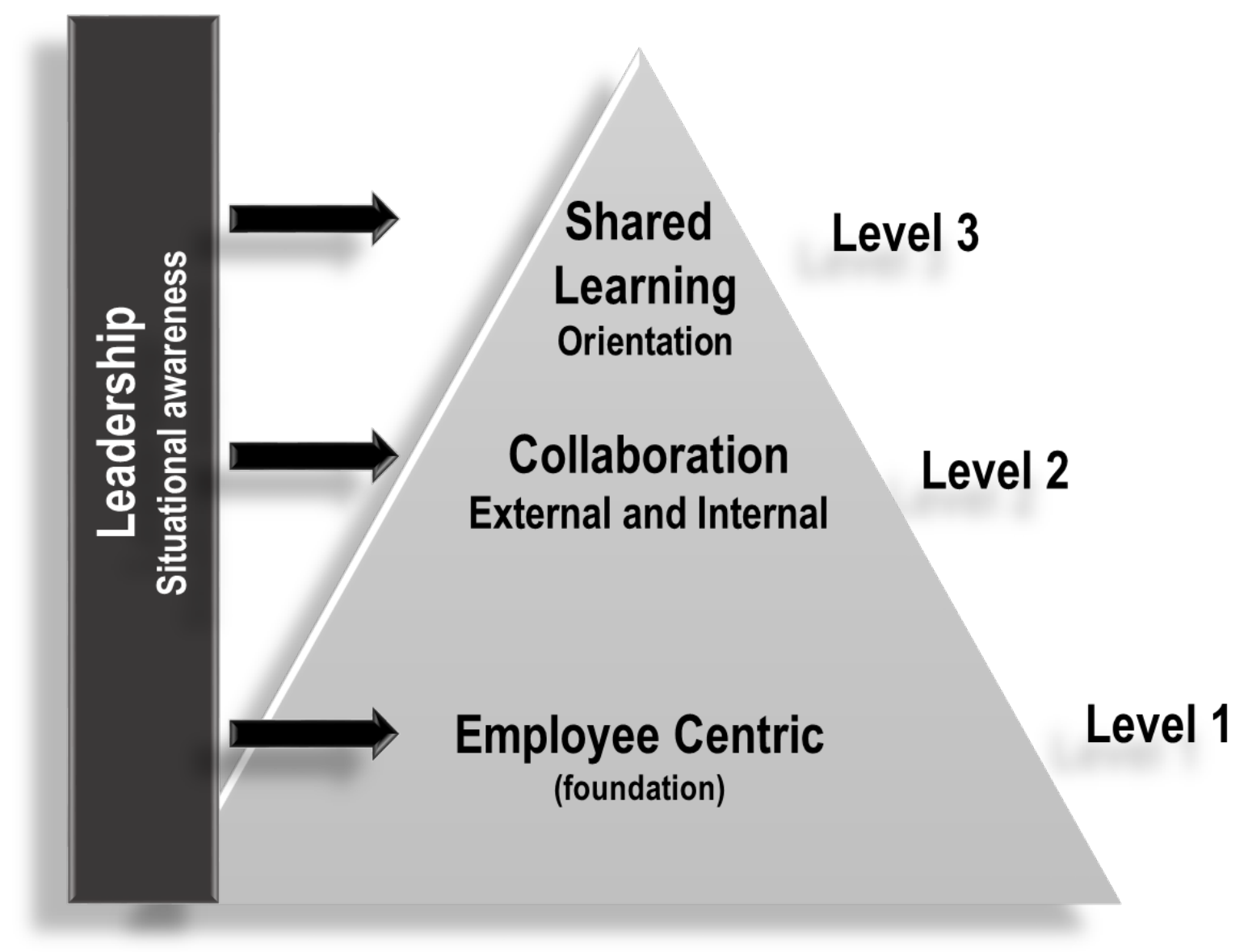

Figure 2. A maturity model of organisational resilience

The longer timeframe and context of this study offers insights into the development of resilience during an extended crisis. While the findings point to specific capacity areas needed for organisational resilience, these are not simple elements that can be changed in a short timeframe. Those key capacities needed to be established in advance, so that they could be drawn upon in a crisis. The more adaptive organisations exhibited cultures that were already employee-centric, collaborative, and supportive of learning before the disaster. In a new, unfamiliar disaster situation, the organisations' ways of operating were based on those existing cultures. The key capacities were utilised from the outset, and in an extended crisis they continued to develop, as part of a trajectory of constantly growing resilience.

In contrast, organisations that evidenced less adaptation had trajectories of slow, late growth. They had limited capability in the four key areas; their leaders lacked situational awareness, and they usually belatedly came to realise the problems caused by their existing, less adaptive approaches. They only moved to develop capacities once the disaster had been in progress for some time.

The maturity model has significant implications for organisations wanting to develop organisational resilience. Viewing the process from this perspective of time-related sequence, progressing from employee-centric orientation through to collaboration to organisational learning, potentially explains why few organisations achieved capacities in all four areas. Consistent with the propositions of earlier writers (de Wall, 2012; Sutcliffe \& 
Vogus, 2003; Vogus \& Sutcliffe, 2007), the study evidences how learning is particularly vital in ambiguous, complex and unfamiliar situations; organisations that are skilled at creating, acquiring, and transferring knowledge show a greater capacity for learning and transformation (for example de Wall, 2012; Sinkula, 1994; Sutcliffe \& Vogus, 2003; Vogus \& Sutcliffe, 2007). However, in the context of a maturity model where organisational learning is the ultimate stage, this requires that the other three areas are already well established, creating the necessary conditions for organisational learning. This then implies that such learning needs to be seen as a very sophisticated competency that takes time to achieve. It is significant to note that each of the key elements typically require much time and work to change; an organisation that lacks these capacities would need to undergo a significant transformation to fully develop organisational resilience, including the higher functions of organisational learning.

The use of paired organisations in the research design indicated that the differences in adaptation could not be solely attributed to factors such as organisation size, or the type of industry. Some of the greatest variation was between two organisations of similar size, in the same sector, with one of the pair demonstrating a markedly higher level of adaptation than the other. Perhaps counterintuitively, an organisation's size was not necessarily an obstacle to resilience; the more adaptive organisations were sometimes large but they identified the need for responsive local leadership and devolved control to situationally aware leaders. Combined with the more developed resilience-related capacities of those organisations, they could move on a path of prompt and effective adaptation.

Finally, the research operationalises a number of existing concepts from other areas of organisational scholarship, combining these to create of model of how resilience develops at an organisational level, in a post-disaster setting. It offers a valuable insight into resilience-inaction. In this respect, the research goes some way towards Britt et al.'s (2016) need for building "more sophisticated models of resilience that conceptualize resilience as a dynamic construct within a dynamic workplace system" (p.394) by introducing a maturity model of resilience in organisations. 


\section{References}

Aldrich, D. P. (2012). Building resilience: social capital in post-disaster recovery. Chicago: The University of Chicago Press.

Ardagh, M., \& Deely, J. (2019). Rising from the Rubble: A health system's extraordinary response to the Canterbury earthquakes New Zealand: Canterbury University Press.

Argote, L., \& Miron-Spektor, E. (2011). Organizational Learning: From Experience to Knowledge. Organization Science, 22(5), 1123-1137.

Beck, T. E., \& Plowman, D. A. (2014). Temporary, Emergent Interorganizational Collaboration in Unexpected Circumstances: A Study of the Columbia Space Shuttle Response Effort. Organization Science, 25(4), 1234-1252. doi:10.1287/orsc.2013.0888

Bhamra, R., Dani, S., \& Burnard, K. (2011). Resilience: the concept, a literature review and future directions. International Journal of Production Research, 49(18), 5375-5393. doi:10.1080/00207543.2011.563826

Boyatzis, R. E., Goleman, D., \& Rhee, K. S. (2000). Clustering Competence in Emotional Intelligence: Insights from the Emotional Competence Inventory. In R. Bar-On, \& Parker, J. D. A. (Ed.), The Handbook of Emotional Intelligence: Theory, Development, Assessment, and Application at Home, School, and in the Workplace. San Francisco, Calif: Jossey-Bass.

Boyatzis, R. E., \& McKee, A. (2005). Resonant Leadership: Renewing Yourself and Connecting with Others Through Mindfulness, Hope, and Compassion: Harvard Business Review Press.

Britt, T. W., Shen, W., Sinclair, R. R., Grossman, M. R., \& Klieger, D. M. (2016). How Much Do We Really Know About Employee Resilience? Industrial and Organizational Psychology, 9(2), 378-404. doi:10.1017/iop.2015.107

Bryant, A., \& Charmaz, K. (2007). Grounded Theory Research: Methods and Practices. In A. Bryant \& K. Charmaz (Eds.), The Sage Handbook of Grounded Theory (pp. 31-57). London: Sage Publications.

Charmaz, K. (2008). Grounded theory as emergent method. In S. N. Hesse-Biber \& P. Leavy (Eds.), Handbook of Emergent Methods (pp. 155-172). New York: The Guilford Press.

Charmaz, K. (2014). Constructing Grounded Theory (Second ed.). London: Sage Publications.

Chen, S., M., W., \& Hobfoll, S. E. (2015). The commerce and crossover of resources: resource conservation in the service of resilience. Stress Health, 31(2), 95-105. doi:10.1002/smi.2574

Christophersen, A., Rhoades, D. A., Hainzl , S., Smith, E. G. C., \& Gerstenberger, M. C. (2013). The Canterbury sequence in the context of global earthquake statistics (2013/196). Retrieved from https://www.eqc.govt.nz/sites/public files/3777-Canterburysequence-in-context-global-earthquake-statistics.pdf

de Wall, A. A. (2012). Characteristics of high performance organzations. Business Management and Strategy, 3(1), 14-31.

Eisenhardt, K. M., \& Graebner, M. E. (2007). Theory building from cases: Opportunities and challenges. Academy of Management Journal, 50(1), 25-32.

Estrada, A., Severt, J., \& Jiménez-Rodríguez, M. (2016). Elaborating on the Conceptual Underpinnings of Resilience. Industrial and Organizational Psychology, 9(2), 497-502. doi:10.1017/iop.2016.46 
Fergusson, D. M., Horwood, L. J., Boden, J. M., \& Mulder, R. T. (2014). Impact of a major disaster on the mental health of a well-studied cohort. JAMA Psychiatry, 71(9), 10251031. doi:doi:10.1001/jamapsychiatry

Gittell, J. H., Cameron, K., Lim, S., \& Rivas, V. (2006). Relationships, Layoffs, and Organizational Resilience: Airline Industry Responses to September 11. The Journal of Applied Behavioral Science, 42(3), 300-329. doi:10.1177/0021886306286466

Hall, C. M., S., M., V., N., R., V., B., W., \& R., W. (2016). Undertaking business, consumer and organisational research in a post-disaster setting. In H. C. M., S. Malinen, R. Vosslamber, \& W. R. (Eds.), Business and Post-disaster Management: Business, organisational and consumer resilience and the Christchurch earthquakes (pp. 251268). Abingdon: Routledge.

Johal, S. S., Mounsey, Z., Brannelly, P., \& Johnston, D. M. (2016). Nurse perspectives on the practical, emotional, and professional impacts of living and working in postearthquake Canterbury, New Zealand. Prehosp Disaster Med, 31(1), 10-16.

Kuntz, J. R. C., Malinen, S., \& Näswall, K. (2017). Employee resilience: Directions for resilience development. Consulting Psychology Journal: Practice and Research, 69(3), 223-242. doi:10.1037/cpb0000097

Kuntz, J. R. C., Näswall, K., \& Malinen, S. (2016). Resilient Employees in Resilient Organizations: Flourishing Beyond Adversity. Industrial and Organizational Psychology, 9(2), 456-462. doi:10.1017/iop.2016.39

Lee, A. V., Vargo, J., \& Seville, E. (2013). Developing a Tool to Measure and Compare Organizations' Resilience. Natural Hazards Review, 14(1), 29-41.

Näswall, K., Malinen, S., \& Kuntz, J. (2017). Resilience Development Through an Organization-Led Well-Being Initiative. In N. Chmiel, F. Fraccaroli, \& M. Sverke (Eds.), An introduction to work and organizational psychology: an international perspective (Third ed., pp. 506-513). Hoboken, NJ: John Wiley \& Sons Inc.

Nilakant, V., Walker, B., Rochford, K., \& van Heugten, K. (2013). Leading in a Post-disaster Setting: A Guide for Human Resource Practitioners. New Zealand Journal of Employment Relations, 38(1), 1-14.

Norris, F. H., Stevens, S. P., Pfefferbaum, B., Wyche, K. F., \& Pfefferbaum, R. L. (2008). Community resilience as a metaphor, theory, set of capacities, and strategy for disaster-readiness. American Journal of Community Psychology, 41, 127-150.

O'Grady, K. A., \& Orton, J. D. (2016). Resilience Processes during Cosmology Episodes: Lessons Learned from the Haiti Earthquake. Journal of Psychology and Theology, 44(2), 109-123. doi:10.1177/009164711604400202

Powley, E. H. (2009). Reclaiming resilience and safety: Resilience activation in the critical period of crisis. Human Relations, 62(9), 1289-1326. doi:10.1177/0018726709334881

Sinkula, J. M. (1994). Market Information Processing and Organizational Learning. Journal of Marketing, 58(1), 35-45.

Stake, R. E. (2000). Case studies. In N. K. Denzin \& Y. S. Lincoln (Eds.), The Handbook of Qualitative Research (2nd ed.): Sage.

Strauss, A., \& Corbin, J. (Eds.). (1997). Grounded Theory in Practice. London: Sage Publications.

Sutcliffe, K., \& Vogus, T. (2003). Organizing for Resilience. In K. S. Cameron, J. E. Dutton, \& R. E. Quinn (Eds.), Positive Organizational Scholarship: Foundations of a New Discipline (pp. 94-110). San Francisco: Berrett-Koehler. 
Tonkin, K., Malinen, S., Näswall, K., \& Kuntz, J. C. (2018). Building employee resilience through wellbeing in organizations. Human Resource Development Quarterly, 29(2), 107-124. doi:10.1002/hrdq.21306

van der Vegt, G. S., Essens, P., Wahlström, M., \& George, G. (2015). Managing risk and resilience, Editorial. Academy of Management Journal, pp. 971-980. Retrieved from http://ezproxy.canterbury.ac.nz/login?url=http://search.ebscohost.com/login.aspx?

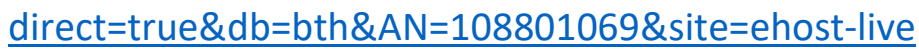

Vanhove, A. J., Herian, M. N., Perez, A. L. U., Harms, P. D., \& Lester, P. B. (2015). Can resilience be developed at work? A meta-analytic review of resilience-building programme effectiveness. Journal of Occupational and Organizational Psychology, n/a-n/a. doi:10.1111/joop.12123

Varker, T., \& Devilly, G. J. (2012). An analogue trial of inoculation/resilience training for emergency services personnel: Proof of concept. Journal of Anxiety Disorders, 26(6), 696-701. doi:https://doi.org/10.1016/j.janxdis.2012.01.009

Vogus, T. J., \& Sutcliffe, K. M. (2007, 7-10 Oct, 2007). Organizational resilience: Towards a theory and research agenda. Paper presented at the IEEE International Conference on Systems, Man and Cybernetics, 2007, ISIC

Wang, C. L., \& Ahmed, P. K. (2003). Organisational learning: a critical review. The Learning Organization, 10(1), 8-17. doi:http://dx.doi.org/10.1108/09696470310457469

Williams, T. A., Gruber, D. A., Sutcliffe, K. M., Shepherd, D. A., \& Zhao, E. Y. (2017). Organizational response to adversity: Fusing crisis management and resilience research streams. Academy of Management Annals, 11(2), 733-769. doi:10.5465/annals.2015.0134

Williams, T. A., \& Shepherd, D. A. (2016). Building Resilience or Providing Sustenance: Different Paths of Emergent Ventures in the Aftermath of the Haiti Earthquake. Academy of Management Journal, 59(6), 2069-2102. doi:10.5465/amj.2015.0682

Yin, R. K. (2003). Case Study Research: Design and Methods (3 ed.). Thousand Oaks, Calif: Sage Publications.

Zollo, M., \& Winter, S. G. (2002). Deliberate learning and the evolution of dynamic capabilities. Organization Science, 13(3), 339-351. 